\title{
Bayesian hierarchical model used to analyze regression between fish body size and scale size: application to rare fish species Zingel asper
}

\author{
B. Fontez ${ }^{(1),(2)}$, L. Cavalli(3),* \\ Received May 24, 2013 \\ Revised February 20, 2014 \\ Accepted February 21, 2014
}

\begin{abstract}
Key-words: hierarchical Bayes model, backcalculation, fish, scales, Zingel asper

Back-calculation allows to increase available data on fish growth. The accuracy of back-calculation models is of paramount importance for growth analysis. Frequentist and Bayesian hierarchical approaches were used for regression between fish body size and scale size for the rare fish species Zingel asper. The Bayesian approach permits more reliable estimation of back-calculated size, taking into account biological information and cohort variability. This method greatly improves estimation of backcalculated length when sampling is uneven and/or small.
\end{abstract}

\section{ABSTRACT}

\section{RÉSUMÉ}

Utilisation d'une approche hiérarchique Bayésienne pour l'analyse de la régression entre la taille du poisson et la taille des écailles : application à l'apron du Rhône, Zingel asper

\author{
Mots-clés : \\ modèles \\ hiérarchiques \\ bayésiens, \\ rétro-calcul, \\ poissons, \\ écailles, \\ Zingel asper
}

\begin{abstract}
Le rétrocalcul permet d'augmenter le nombre de données disponibles pour l'analyse de la croissance des poissons. La précision des modèles de régression est cruciale pour l'analyse de la croissance. Deux approches hiérarchiques, Bayésienne et fréquentiste, sont proposées pour l'analyse de la régression entre la taille du poisson et la taille de l'écaille, en prenant pour exemple, une espèce rare, l'apron Zingel asper. L'approche Bayésienne hiérarchique donne une estimation plus fiable de la taille rétrocalculée des poissons pour des échantillons de petite taille, en prenant en compte des informations biologiques sur l'espèce et la variabilité au sein des cohortes. Cette méthode améliore nettement l'estimation de la longueur rétrocalculée dans le cas d'échantillonnages réduits ou mal distribués.
\end{abstract}

Estimating fish growth rates is fundamental in fisheries biology (Bagenal and Tesch, 1978; Francis, 1990) and for the management of endangered species (Akçakaya and Sjögren-Gulve, 2000). Back-calculation from scales or otoliths has been used for decades, allowing to increase available data on fish growth (Francis, 1990) especially for rare species, where it is generally unavailable. Back-calculation supposes a relation between both fish and scale (or otolith) lengths for each cohort of a population. The regression model approximating this

(1) Montpellier SupAgro, UMR MISTEA, 34060 Montpellier Cedex 2, France

(2) INRA, UMR0729 MISTEA, 34060 Montpellier Cedex 2, France

(3) Aix Marseille Université, UMR7263 IMBE, Institut Méditerranéen de Biodiversité et d'Ecologie, Équipe Ecologie des Eaux Continentales, 1 pl V Hugo, 13001 Marseille, France

* Corresponding author: laurent.cavalli@imbe.fr 


\section{Table I}

Number of fish analyzed on the Durance population, cohorts 1997 to 2004.

\begin{tabular}{|c|c|c|c|c|c|}
\cline { 2 - 6 } \multicolumn{1}{c|}{} & $0+$ & $1+$ & $2+$ & $3+$ & $4+$ \\
\hline $\mathbf{1 9 9 7}$ & 0 & 0 & 95 & 20 & 3 \\
\hline $\mathbf{1 9 9 8}$ & 0 & 131 & 57 & 7 & 0 \\
\hline $\mathbf{1 9 9 9}$ & 6 & 31 & 17 & 0 & 0 \\
\hline $\mathbf{2 0 0 0}$ & 0 & 29 & 1 & 1 & 1 \\
\hline $\mathbf{2 0 0 1}$ & 20 & 22 & 27 & 9 & 0 \\
\hline $\mathbf{2 0 0 2}$ & 4 & 34 & 14 & 0 & 1 \\
\hline $\mathbf{2 0 0 3}$ & 77 & 22 & 12 & 5 & 2 \\
\hline $\mathbf{2 0 0 4}$ & 0 & 11 & 8 & 0 & 0 \\
\hline
\end{tabular}

relation for the lengths at capture is then used to infer individual growth. Various authors raise the issue of potential biases in the estimation due to inter-cohort growth variability, age or nonlinear effects (Campana, 1990; Morita and Matsuishi, 2001; see Wilson et al., 2009 for a review). To take into account intercohort variability, using only one regression for the whole population is inappropriate. But one regression per cohort requires large, wellbalanced samples. When sampling is uneven or small, hierarchical models allow information on different cohorts to be shared, thus reinforcing the cohort base and leading to more accurate estimation (Gelman and Hill, 2007; Pinheiro and Bates, 2001). To reduce biases in the estimation, Campana (1990) proposed a biological intercept model using biological information at hatching instead of regression. Next, Vigliola et al. (2000) included this biological information in the regression, fixing the intercept to biological value, as a modified Fry model (Fry, 1943). The Bayesian approach can integrate a priori biological information in addition to information obtained from measurements; this approach is sufficiently flexible to fit hierarchical models. Bayesian methods, frequently used in ecology (Royle and Dorazio, 2008; Parent and Rivot, 2012), allow flexible modeling of length observations from capture-recapture data (Zhang et al., 2009; Sigourney et al., 2012; Schofield et al., 2013). They are not usually applied to back-calculation computation, although they are easy to implement using $R$ (see for example Gelman and Hill, 2007; package Imer source, http://r-forge.r-project.org/projects/lme4/) or WinBUGS/OpenBUGS (Lunn et al., 2000 or Ntzoufras, 2009; http://www.mrc-bsu.cam.ac. uk/bugs/winbugs/contents.shtml) Here, a low-density endangered fish species Zingel asper (L.) on which little data is available was used to assess the performance of a classic linear model and hierarchical models in both frequentist and Bayesian formulations. Given the short lifespan of this species nonlinear and age effects were ignored. Growth was supposed homogenous within a cohort and variable between cohorts The models are presented and we identify conditions where the Bayesian hierarchical approach is more accurate.

Zingel asper (L.) is cited as endangered in the Habitats Directive of the European Commission, Annexes II and IV, and in the Bern Convention, Annex II, and critically endangered in the IUCN red list of threatened species (http://www.iucnredlist.org/details/23207/0). Sampling points were positioned on a segment of the Durance River, from Tallard to Sisteron (between 14.5 and 32.8 kilometers downstream of the Tallard dam), closed off by impassable dams. 1284 individuals belonging to the same population were collected from 1999 to 2007 (Table I) using electrofishing equipment (Héron, Dream Electronic Equipment, Generator, DC, 300V, 3A, one anode). Each fish caught was measured (fork length, $L f$ ) to the nearest $\mathrm{mm}$; scales were removed for age determination (Cavalli et al., 2003). 99\% of the fish were aged from 0+ to 4+. The few older fish were discarded because age could not be determined with certainty.

First, the quadratic model proposed by Danancher et al. (2007) for $Z$. asper was used:

Model 1

$$
L f_{i j}=\beta_{o j}+\beta_{1 j} L s_{i j}+\beta_{2} L s_{i j}^{2}+\varepsilon_{i j},
$$

where error terms $\varepsilon_{i j}$ are independent and identically distributed (iid) $\varepsilon_{i j} \sim{ }_{i i d} N\left(0, \sigma^{2}\right)$; $L f_{i j}$ is the fork fish length and $L s_{i j}$, is the scale length for fish $i$ in cohort $j$. Between cohorts, growth is supposed variable, so one equation for each cohort $j$ was fitted. 


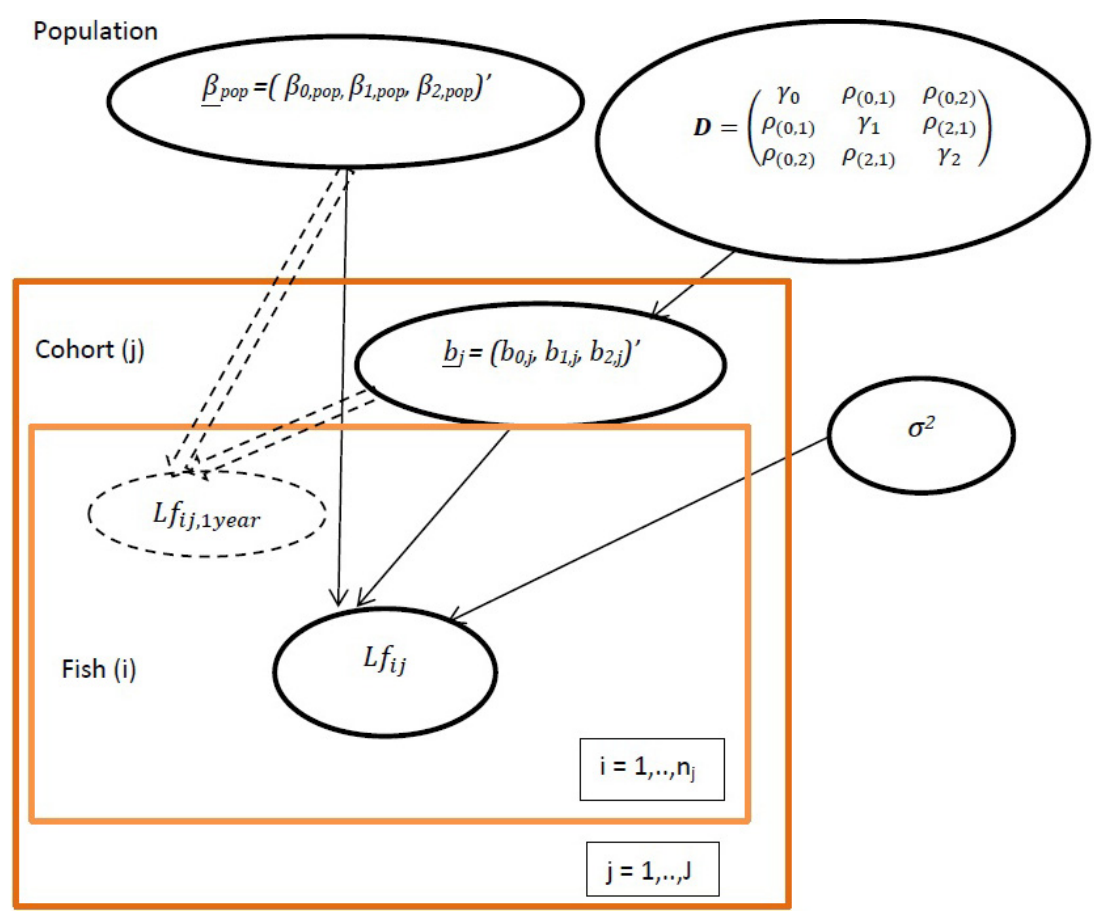

\section{Figure 1}

Directed Acyclic Graph: representation of the Bayesian hierarchical quadratic model for the Z. asper example. Stochastic nodes (random variables) are denoted by ellipses. Solid arrows indicate stochastic dependence whereas hollow arrows indicate logical function. Repeated parts are represented using plates. Eventually, the dashed elements correspond to the addition of a latent variable (back-calculated size at one year old). ( $L f$ fork fish length at capture; $L f_{1}$ year, back calculated fork fish length at one year.)

To share cohort information, a mixed model (belonging to the class of hierarchical models) was used, with cohort as random effect:

Model 2

$$
L f_{i j}=\left(\beta_{0 p o p}+b_{0 j}\right)+\left(\beta_{1 p o p}+b_{1 j}\right) L s_{i j}+\left(\beta_{2 p o p}+b_{2 j}\right) L s_{i j}^{2}+\varepsilon_{i j}
$$

where $\varepsilon_{i j} \sim{ }_{i i d} N\left(0, \sigma^{2}\right)$ independent of $\underline{b}_{j}=\left(b_{o j}, b_{1 j}, b_{2 j}\right)^{\prime} \sim \sim_{i i d} N_{3}(\underline{0}, \boldsymbol{D})$

All cohorts belong to the same population and have in common the vector $\beta_{\text {pop }}=\left(\beta_{0 \text { pop }}\right.$, $\left.\beta_{1 p o p,} \beta_{2 p o p}\right)^{\prime}$, the mean regression factor for all cohorts, which can be considered as the vector of regression factors for the population mean curve. In contrast, each cohort has its own variability and the term $\underline{b}_{j}$ represents the discrepancy between cohort $j$ and mean population curve. For all cohorts, a common unspecified variance-covariance matrix, $\boldsymbol{D}$, models the variability of and the dependencies between latent variables $\left(b_{o j}, b_{1 j}, b_{2 j}\right)$. The model was estimated using R package Ime4 (function Imer). The program is detailed in the supporting file.

In order to improve our estimation, $Z$ asper a priori biological information was included in our third model using a Bayesian approach.

Model 3

$$
\begin{aligned}
& L f_{i j}=\left(\beta_{0 p o p}+b_{0 j}\right)+\left(\beta_{1 p o p}+b_{1 j}\right) L s_{i j}+\left(\beta_{2 p o p}+b_{2 j}\right) L s_{i j}^{2}+\varepsilon_{i j} \\
& \text { With } \varepsilon_{i j} \sim{ }_{i i d} N\left(0, \sigma^{2}\right) \text { independent of } \underline{b}_{j}=\left(b_{0 j}, b_{1 j}, b_{2 j}\right)^{\prime} \sim_{i i d} N_{3}(\underline{0}, \boldsymbol{D}) \\
& \underline{\beta}_{\text {pop }} \sim N_{3}(\underline{m}, \boldsymbol{V}), \sigma^{2} \sim I G\left(\text { shape, rate) and } \boldsymbol{D} \sim I W\left(d, \boldsymbol{R}^{-1}\right)\right.
\end{aligned}
$$

The terms in (3.3) allowed us to specify a priori information on parameters $\underline{\beta}_{p o p}, \boldsymbol{D}$ and $\sigma^{2}$ (defined in model 3 and on the DAG Figure 1). We used two sources of biological information, a bibliographic reference (Danancher et al., 2007) on the Beaume river and unpublished data on another population on the Durance river (Salignac) close to the studied population. 
Since $Z$. asper was spread over a limited geographic area and ecological conditions for the Beaume and Durance populations were similar, we chose bibliographic references for $\beta_{p o p}$. However, Danancher et al. (2007) did not give dependences between parameters ( $\boldsymbol{V}$ and $\boldsymbol{D}$ variance-covariance matrices), and therefore personal unpublished data (Salignac population) were used. Model 3 with vague prior and model 2 were applied to Salignac data. We used the estimates of $\boldsymbol{V}$ from both models and posterior estimate of $\boldsymbol{D}$ to define the prior information for the Durance population.

$$
-\underline{\beta}_{p o p}: \text { we considered } \underline{m}=(1.67,7.46,-0.67) \text { and } \boldsymbol{V}=\left(\begin{array}{ccc}
0.2 & -0.3 & 0.1 \\
-0.3 & 0.5 & -0.2 \\
0.1 & -0.2 & 0.07
\end{array}\right) \text {. }
$$

- D : Inverse Wishart (IW) (Robert, 2001) is a distribution law for the variance-covariance matrix $\boldsymbol{D}$ with $\boldsymbol{R} /(\boldsymbol{d}-2)$ as average (see Eq. 3.3). This distribution is often used due to its conjugate propriety with the normal distribution. Based on the Salignac data, we considered a diagonal matrix $\boldsymbol{R}=\boldsymbol{I}_{3}$. Next, to ensure a well covering of the cohort latent variables' space, the a priori average of $\boldsymbol{D}$ was set to larger values $\left(10^{-2} \boldsymbol{I}_{\mathbf{3}}\right)$ than what was observed a posteriori at Salignac $\left(10^{-4} / 3\right)$, by setting $d=100$.

- $\sigma^{2}$ : an Inverse Gamma (IG) (Robert, 2001) was used for conjugate commodity and to ensure positive values for this variance term. IG parameters were defined in such a way as to take account of the uncertainty of the fish length's measurement at capture (to the nearest $\mathrm{mm}$ ). Consequently, we defined the two parameters of the inverse gamma as shape $=10$ and rate $=$ 0.1 , in order to obtain an expected value, $E\left(\sigma^{2}\right)=1 \mathrm{~mm}$.

Bayesian estimation is made on the posterior distribution $p\left(\underline{b}_{1}, \ldots, \underline{b}_{8}, \underline{\beta}_{p o p}, \sigma^{2}, \boldsymbol{D} / \underline{L f}\right)$. Samples of this posterior distribution can be obtained by sampling sequentially from all the conditional posterior distributions: $p\left(\underline{b}_{1} / \underline{b}_{2}, \ldots, \underline{b}_{8}, \underline{\beta}_{p o p}, \sigma^{2}, \boldsymbol{D}, \underline{L f}\right), \ldots, p\left(\boldsymbol{D} / \underline{b}_{1}, \ldots, \underline{b}_{8}, \underline{\beta}_{\text {oop }}, \sigma^{2}, \underline{L f}\right)$, (Gibbs sampler, Robert \& Casella, 1999). It meant for $Z$. asper example that each level of Figure 1 was sampled in turn. Estimates were defined as the means of this sampling computed with WinBUGS (40 000 samples after 1000 burn-in samples with no thinning, see supporting file). Bayesian models were built using both the informative prior given above and a less informative prior. This less informative prior was not really a vague prior, which refers to a uniform law where each value has the same probabilty of being observed. In our case, $\boldsymbol{D}$ and $\boldsymbol{V}$ values were multiplied by 100 to obtain a flat curve for $\underline{\beta}_{p o p}$ and $\underline{b}_{j}$ normal distribution. To compare the two priors (less informative and informative), we computed the posterior mean and variance for each observation (Carlin and Louis, 2000). Then, the residual sum of squares (goodness of fit) and the sum of the posterior variances were the two criteria used for model assessment. Using the Bayesian approach, the back-calculated length was directly estimated. This is a latent variable and therefore can be estimated like the other model variables, through sampling from the posterior distribution (see Bayesian model, Figure 1 and supporting file). Autocorrelation between successive samples of a variable (parameter or latent variable) was low (less than 0.15 ) or negligible depending on the variable. Therefore, all the 40000 samples were used without thinning. While not shown, trace plots showed good mixing and we assumed that the chain had converged to the posterior distribution. Density estimates appeared unimodals and relatively smooth (Figure 2), suggesting that Gibbs sampler was computationally efficient. Eventually, the Geweke (1992) test statistics was in favor of the chain convergence. When the quadratic fixed effects model (Model 1) was fitted to each cohort of Z. asper independently, cohorts without young fish (mainly 1997 and 2004) were poorly estimated (Figure 3). The mixed frequentist model (Model 2) gave better estimations with this uneven sampling. Of the Bayesian models, the informative model gave better results than the less informative model, with respectively goodness of fit values 656 and 720 and sum of posterior predictive variances 1306 and 1496. Comparisons between the informative Bayesian and frequentist mixed models showed differences (Table II and Figure 3). Regression curves obtained using the Bayesian model better reflect biological knowledge of fish growth, with a growth increment decrease after sexual maturity (at age 3 for $Z$. asper), than does the frequentist mixed model. Moreover, with the Bayesian model, posterior distribution of the mean 


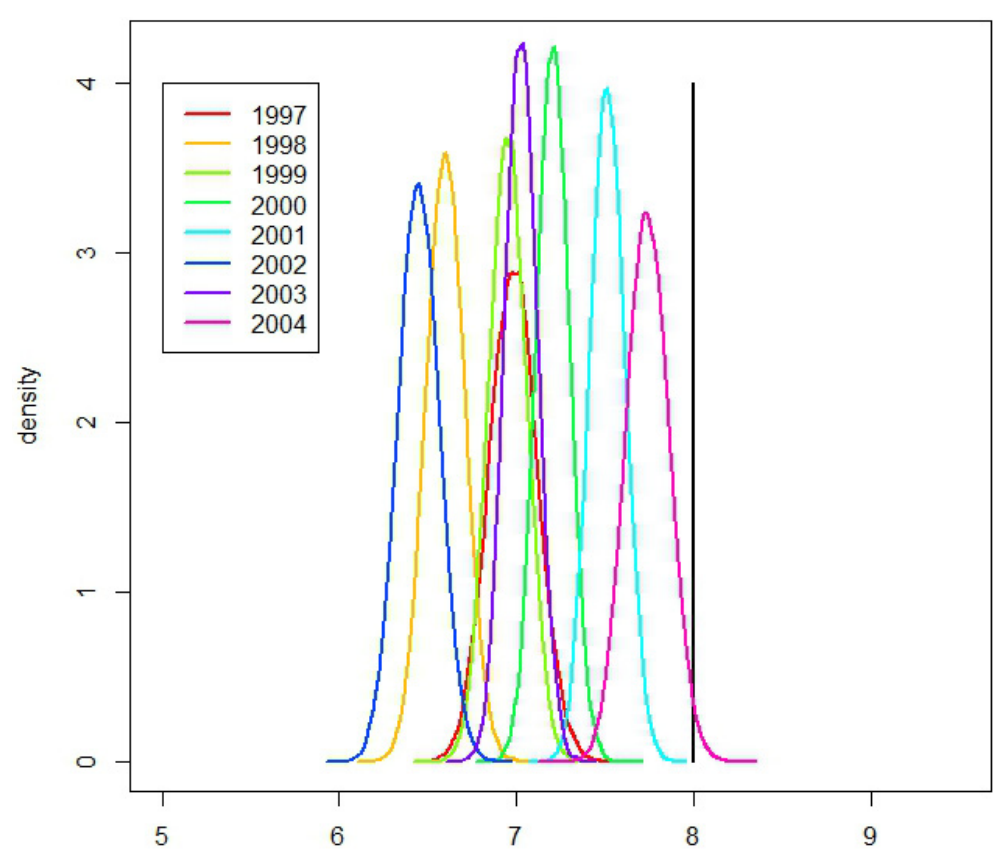

Figure 2

Kernel density estimates of the marginal posterior density of the mean back-calculated length at 1 year for the different cohorts of the Durance population (1997 to 2004), using the Bayesian mixed model with informative prior.

back-calculated length at 1 year can be obtained for each cohort (Figure 2) with information both on the distribution of length (position and dispersion) for each cohort. Figure 2 showed that the posterior marginal probability of the mean of each cohort's back-calculated length at 1 year being greater than $8 \mathrm{~cm}$ is near 0 on the Durance river.

Regression of body length on scale (otolith) size is rarely detailed in the literature. However, this regression is essential, and incorrect estimation would induce biased growth results for back-calculated lengths. Thus, when sampling is unbalanced between cohorts (or populations) or when the sample size is too small, as is often the case with rare species, parameter estimation performed with cohort regressions might be misleading, yielding unreliable values (as for Z. asper Figure 3). Frequentist or Bayesian mixed models allow for the adjustment of multiple cohorts and can reduce the effect of unbalanced samples. As underlined by Zhang et al. (2007), a hierarchical Bayesian approach can remain robust. Moreover, the informative prior may compensate for lack of data. The Bayesian approach allows for a better estimation when biological information is available. For example, $\underline{\beta}_{\text {pop }}$ parameters can be considered correlated (Weatherley and Gill, 1987). Taking into account these correlations using the variance covariance matrix $\boldsymbol{V}$ in the Bayesian model allowed us to obtain a more accurate estimation of fish growth than with a frequentist model. Danancher et al. (2007) observed back-calculated lengths at 1 year greater than $8 \mathrm{~cm}$. Based on the hierarchical Bayesian model with informative prior, scalation length appears greater on the Durance river, while back-calculated length at 1 year is less than in the Beaume river (Danancher et al., 2007). These results agree with those of Cavalli et al. (2003), showing a shorter optimal growth period and a cooler water temperature during spring and summer in the Durance river than in the Beaume river. However, the hyperparameter $\underline{m}$, whose value was fixed from Beaume river data (Danancher et al., 2007), has no influence on final results. In the case studied, the model is not sensitive to this variable. Generally, however, when using the Bayesian model, it is important to analyze sensitivity to the a priori biological information.

This study addressed growth history as coded in scales or otoliths, focusing on fish length because it represents a meaningful working variable. Another approach is to use scale or otolith length directly to study fish growth (Alos et al., 2010; Weisberg et al., 2010). However, 


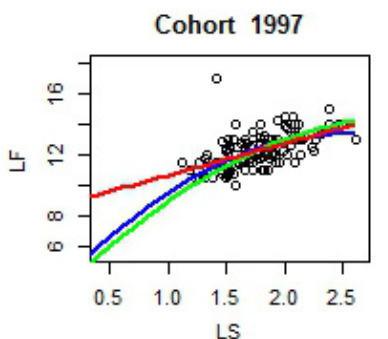

LS
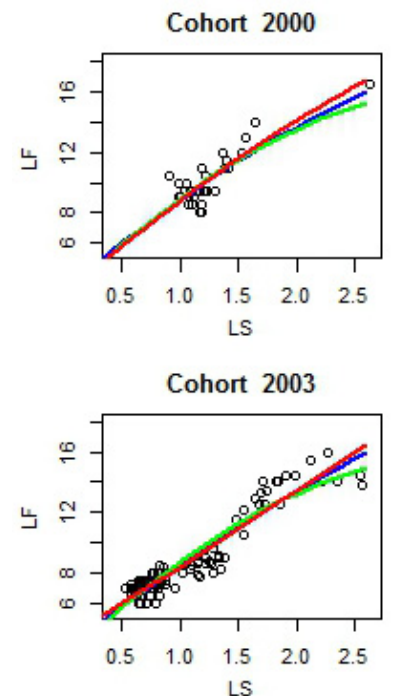

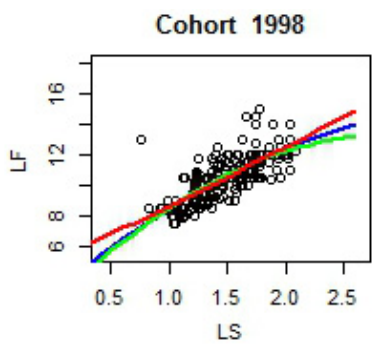

Cohort 2001

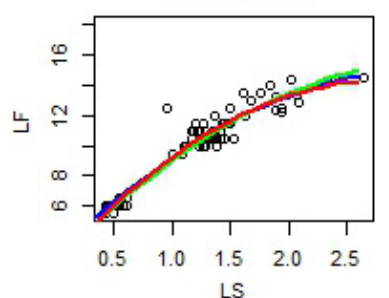

Cohort 2004

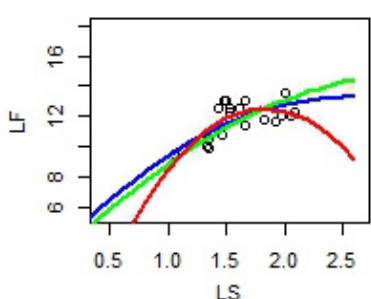

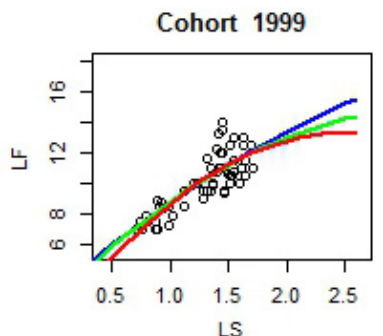

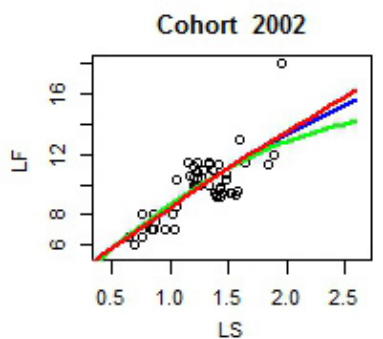

\section{Figure 3}

Scatter plot of cohort fish length data with adjusted quadratic linear regression, from respectively the cohort fixed effects model (red), the frequentist and Bayesian mixed models (respectively blue and green). (LS, scale length ; LF, fork fish length).

\section{Table II}

$\underline{\hat{\beta}}_{\text {pop }}$ ( \pm standard errors) and $\underline{\hat{b}}_{\text {j }}$ estimates for frequentist and Bayesian informative mixed models.

\begin{tabular}{|c|c|c|}
\cline { 2 - 3 } \multicolumn{1}{c|}{} & \multicolumn{2}{c|}{ Frequentist mixed model Durance } \\
\cline { 2 - 3 } \multicolumn{1}{c|}{} & $\underline{\hat{\beta}}_{\text {pop }}$ & $\hat{\underline{\beta}}_{\text {pop }}+\underline{\underline{b}}_{j} j$ in $\{1997, \ldots, 2004\}$ \\
\hline$\beta_{0}$ & $2.94 \pm 0.44$ & $\{2.30,4.40,2.97,2.51,2.19,3.27,3.50,2.41\}$ \\
\hline$\beta_{1}$ & $6.77 \pm 0.84$ & $\{8.88,4.27,6.22,6.99,8.48,5.54,4.98,8.81\}$ \\
\hline$\beta_{2}$ & $-0.83 \pm 0.34$ & $\{-1.79,-0.1,-0.49,-0.71,-1.47,-0.24,-0.02,-1.8\}$ \\
\hline
\end{tabular}

\begin{tabular}{|c|c|c|}
\cline { 2 - 3 } \multicolumn{1}{c|}{} & \multicolumn{2}{|c|}{ Bayesian mixed model Durance } \\
\cline { 2 - 3 } \multicolumn{1}{c|}{} & $\underline{\hat{\beta}}_{\text {oop }}$ & $\underline{\hat{\beta}}_{\text {oop }}+\underline{\underline{b}}_{j} j$ in $\{1997, \ldots, 2004\}$ \\
\hline$\beta_{0}$ & $2.23 \pm 0.29$ & $\{2.37,2.15,2.21,2.23,2.33,2.19,2.14,2.27\}$ \\
\hline$\beta_{1}$ & $7.73 \pm 0.42$ & $\{7.82,7.62,7.72,7.76,7.81,7.69,7.68,7.75\}$ \\
\hline$\beta_{2}$ & $-0.67 \pm 0.16$ & $\{-1.25,-1.29,-1.16,-1.06,-1.13,-1.18,-1.06,-1.18\}$ \\
\hline
\end{tabular}

as Alos et al. (2010) point out, interpretation is facilitated by using fish length instead of otolith length. It would therefore appear worthwhile to extend the Bayesian model (Model 3 ) to build a comprehensive model for fish size increment and growth analysis which would take into account extrinsic and intrinsic growth factors as suggested by Weisberg et al. (2010), Helser et al. (2012) or models that account for individual variation while taking into account uncertainty in measurements (Sigourney et al., 2012). In our study, uncertainty in measurements was not taken into account because of the short $Z$. asper lifespan, but scale erosion or age determination errors can be integrated into the Bayesian model as suggested by Sigourney et al. (2012). 


\section{ACKNOWLEDGEMENTS}

The authors wish to thank all those who participated in the fish sampling, especially $R$. Chappaz (IMBE), and P. Moullec (ONEMA-SD05). We are grateful to Marjorie Sweetko for improving the English of the manuscript. The authors also wish to acknowledge P. Vuillaume who corrected our written English.

\section{REFERENCES}

Akçakaya H.R. and Sjögren-Gulve P., 2000. Population viability analyses in conservation planning: an overview. Ecol. Bul., 48, 9-21.

Alos J., Palmer M., Alonso-Fernandez A. and Morales-Nin B., 2010. Individual variability and sex-related differences in the growth of Diplodus annularis (Linnaeus, 1758). Fish. Res., 101, 60-69.

Bagenal T.B. and Tesch F.W., 1978. Age and Growth. In: Bagenal T.B. (ed.), Methods for the assessment of fish production in fresh waters. IBP Handbook No 3 (3rd edn). Blackwell, Oxford, 365 p.

Campana S.E., 1990. How reliable are growth back-calculation based on otoliths? Can. J. Fish. Aquat. Sci., 47, 2219-2227.

Carlin B.P. and Louis T.A., 2000. Bayes and Empirical Bayes methods for data analysis. In: Texts in statistical science. Chapman \& Hall, New York, $419 \mathrm{p}$.

Cavalli L., Pech N. and Chappaz R., 2003. Diet and growth of the endangered Zingel asper in the Durance river. J. Fish Biol., 63, 460-471.

Danancher D., Labonne J., Gaudin P. and Joly P., 2007. Scales measurements as a conservation tool in endangered Zingel asper (Linnaeus, 1758). Aquat. Conserv.: Mar. Freshwat. Ecosyst., 17, 712-723.

Francis R.I.C.C., 1990. Back-calculation of fish length: a critical review. J. Fish Biol., 36, 883-902.

Fry F.E.J., 1943. A method for the calculation of the growth of fishes from scale measurements. Publ. Ont. Fish Res. Lab., 61, 5-18.

Gelman A. and Hill J., 2007. Data Analysis Using Regression and Multilevel/Hierarchical Models. Cambridge University Press, $610 \mathrm{p}$.

Geweke J., 1992. Evaluating the accuracy of sampling-based approaches to the calculation of posterior moments. In: Bayesian Statistics 4, Oxford University Press, $876 \mathrm{p}$.

Helser T.E., Lai H-L. and Black B.A., 2012. Bayesian hierarchical modeling of pacific geoduck growth increment data and climate indices. Ecol. Model., 247, 210-220.

Lunn D.J., Thomas A., Best N. and Spiegelhalter D., 2000. WinBUGS - a Bayesian modelling framework: concepts, structure, and extensibility. Stat. Comput., 10, 325-337.

Morita K. and Matsuishi T., 2001. A new model of growth back-calculation incorporating age effect based on otoliths. Can. J. Fish. Aquat. Sci., 58, 1805-1811.

Ntzoufras I., 2009. Bayesian modeling using WinBUGS: An Introdution. Wiley Series in Computational Statistics, $520 \mathrm{p}$.

Parent E. and Rivot E., 2012. Introduction to Hierarchical Bayesian Modeling for Ecological Data. CRC Press, Boca Raton, $405 \mathrm{p}$.

Pinheiro J.C. and Bates D.M., 2001. Mixed Models in S and S-Plus, 2nd edn, NY: Springer Verlag, 530 p.

Robert C.P., 2001. The Bayesian choice, 2nd edn. Springer texts in statistics, New York, Springer-Verlag, $605 \mathrm{p}$.

Robert C.P. and Casella G., 1999. Monte Carlo statistical methods. Springer texts in statistics, New York, Springer-Verlag, $420 \mathrm{p}$.

Royle J.A. and Dorazio R.M., 2008. Hierarchical Modeling and Inference in Ecology: the Analysis of Data from Populations, Metapopulations and Communities. Academic Press, New York, 445 p.

Schofield M.R., Barker R.J. and Taylor P., 2013. Modelling individual specific fish length from capturerecapture data using the von Bertalanffy growth curve. Biometrics, 69, 1012-1021.

Sigourney D.B., Munch S.B. and Letcher B.H., 2012. Combining a Bayesian nonparametric method with a hierarchical framework to estimate individual and temporal variation in growth. Ecol. Model., 247, 125-134. 
Vigliola L., Harmelin-Vivien M. and Meekan M.G., 2000. Comparison of techniques of back-calculation of growth and settlement marks from the otoliths of three species of diplodus from the Mediterranean Sea. Can. J. Fish. Aquat. Sci., 57, 1291-1299.

Weatherley A.H. and Gill H.S., 1987. The biology of fish growth. Academic Press, London, $443 \mathrm{p}$.

Weisberg S., Spangler G. and Richmond L.S., 2010. Mixed effects models for fish growth. Can. J. Fish. Aquat. Sci., 67, 269-277.

Wilson J.A., Vigliola L. and Meekan M.G., 2009. The back-calculation of size and growth from otolith: Validation and comparison of models at an individual level. J. Exp. Mar. Biol. Ecol., 368, 9-21.

Zhang Z.Y., Hamagami F., Wang L.J., Nesselroade J.R. and Grimm K.J., 2007. Bayesian analysis of longitudinal data using growth curve models. Int. J. Behav. Dev. 31, 374-383.

Zhang Z.Y., Lessard J. and Campbell A., 2009. Use of Bayesian hierarchical models to estimate northern abalone, Haliotis kamtschatkana, growth parameters from tag-recapture data. Fish. Res., 95, 289-295. 